\title{
M-estimation of Boolean models for particle flow experiments
}

\author{
Jason A. Osborne \\ Department of Statistics, North Carolina State University, Raleigh, NC, USA \\ Tony E. Grift \\ Department of Agricultural and Biological Engineering, University of Illinois, Urbana, IL, USA
}

\begin{abstract}
Summary. Probability models are proposed for passage time data collected in experiments with a device designed to measure particle flow during aerial application of fertilizer. Maximum likelihood estimation of flow intensity is reviewed for the simple linear Boolean model, which arises with the assumption that each particle requires the same known passage time. M-estimation is developed for a generalization of the model in which passage times behave as a random sample from a distribution with a known mean. The generalized model improves fit in these experiments. An estimator of total particle flow is constructed by conditioning on lengths of multi-particle clumps.
\end{abstract}

\section{Introduction}

Measuring the outflow of granular particles from an airborne spreader during the aerial application of fertilizer or pesticide presents agricultural engineers with a difficult problem. The goal of uniform distribution over a targeted field requires knowledge about flow rate of the material as it is dropped from the aircraft. Windspeed, air speed, granule properties, humidity, and temperature have been identified (Casady et al. (1997)) as factors which can lead to variability in these outflow rates and hence amounts of material that reach the target. Typically, applicators are calibrated annually so that they achieve an average target flow rate. In practice, pilots use a simple lever-operated gate to change the flow rate in order to account for extreme values of these factors. This adjustment is based on intuition, without any feedback from measurement of particle flow.

One approach to providing the pilot with more information uses an optical sensor device (Grift and Hofstee (1997)) which measures the velocity (in meters per second) and size of clumps of particles as they flow through the spreader duct. This device has two photo-sensitive arrays of optical sensors that receive a signal from a light source. As a particle passes an active area, it blocks this light thereby interrupting the signal received by the sensors. As long as all of the sensors in the array are receiving a high signal, the channel is classified as unoccupied and this is taken as an indication that there are no particles flowing through at that instant. If the signal to any one of sensors is interrupted, this is interpreted as the presence of at least one particle, constituting a clump, in flow. The two sensor arrays are 0.00078 meters apart and it is possible to measure the time in seconds that it takes a clump to move from one array to the other, $\Delta t_{f}$. The total time that either array is blocked, $\Delta t_{b}$, is also measured, facilitating calculation of velocity in meters per second, $v=0.00078 / \Delta t_{f}$ and clump length in meters, $C L=v \Delta t_{b}$. These observable clump lengths, either in terms of physical length in meters or time in seconds, are the basis for inference about particle flow in the system. Such a measurement device is called a type II counter (Pyke (1958)).

Grift et al. (2001) and Grift (2002) carried out bench-scale experiments to evaluate the optical sensor device in situations designed to simulate the flow of fertilizer particles through an airborne spreader duct. In these experiments, a known number of spherical particles with a known mean diameter of $4.45 \mathrm{~mm}$ was dropped from predetermined heights through a duct on which the sensor device was installed. The heights from which the particles were dropped was controlled at several 
Run \#20

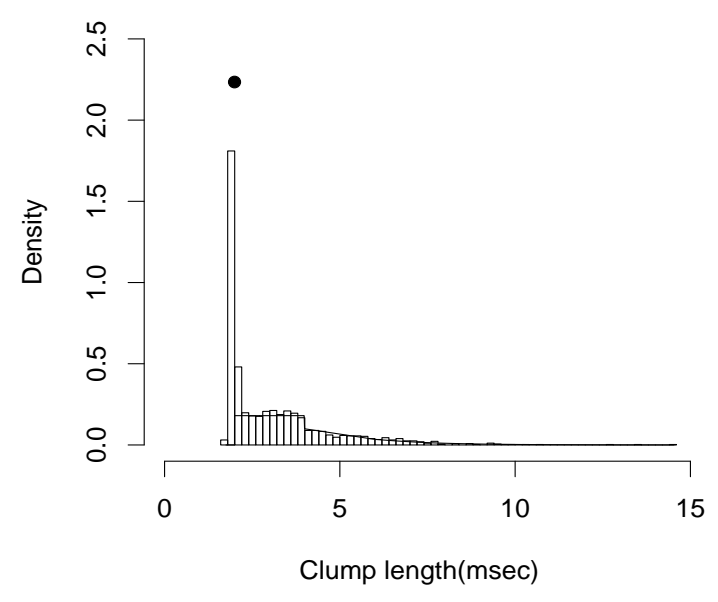

Fig. 1. Probability histogram of $N(t)=1790$ clump lengths in $m s e c$

values to simulate a range of particle velocities and flow rates. A histogram representing the distribution of particle clump lengths in units of time, obtained from one run of these experiments, is shown in Figure 1. The relative frequencies for clump lengths (in $m s e c$ ) are based on dropping 4000 spherical steel particles (actually BBs) from a fixed height.

In this paper, simple linear Boolean models are used to describe the clump length data generated by the Grift experiments, thus providing a basis for inference about flow rates and total particle flow during a dispersal period. In particular, flow intensity is quantified by a single rate parameter in a simple Boolean model. Maximum-likelihood is reviewed in cases where particles require a fixed time for passage and an $M$-estimator is obtained in more general cases. Assessment of total particle flow utilizing this estimator is also developed.

Section 2 introduces the Boolean models and establishes notation and terminology. Results for the clump length distribution derived in Hall (1988) are used to develop $M$-estimation of flow intensity and the $M$-estimator is compared with maximum likelihood and other moment estimators by simulation. In section 3 , two estimators of total particle flow are proposed, including one obtained by derivation of the conditional expectation of the number of particles in a clump given clump length under the equal diameters model. Simulations are carried out to give an assessment of the performance of this estimator in the random passage times model. The methods are evaluated based on their performance with the experimental data in Section 4. Section 5 concludes.

\section{Estimation}

To obtain a probability model for the clump-length data, particles are assumed to be identically spherical with a known diameter, $d_{0}$, and to arrive at the sensor according to a homogeneous Poisson process with unknown intensity $\lambda$. Passage of particles is assumed to continue unabated upon arrival at the sensor. In one version of the model, the particles are travelling at a constant velocity, say $v_{0}$, and the segment length (Hall (1988)), or time required for any single particle to pass the sensor, is constant at $t_{0}=d_{0} / v_{0}$. In a second version, velocities or diameters are assumed to vary in such a way that segment lengths behave as a random sample from a population with a known mean $\mu$ and 


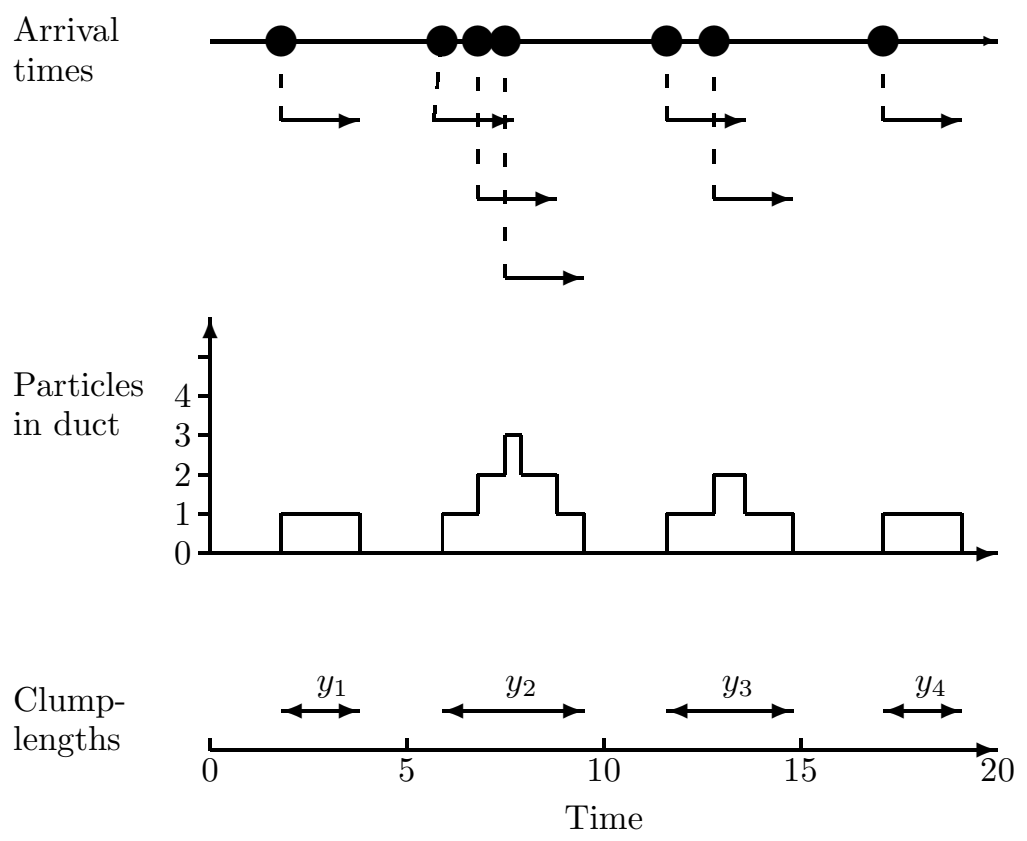

Fig. 2. A diagram of particle flow measurement using a type II counter

an unknown variance $\sigma^{2}$. The two models will be referred to as deterministic segment length (DSL) or random segment length (RSL) models, respectively.

Suppose that particle flow is observed for $t$ time units. Let the number of particles arriving at the sensor in this time period be denoted by $A(t)$. Let $N(t)$ denote the number of complete particle clumps observed by time $t$. Let $Y_{1}, Y_{2}, \ldots, Y_{N(t)}$ denote the lengths of these clumps and $Z_{1}, Z_{2}, \ldots$ the spacings between them. Let the unobservable number of particles comprising clump $i$ be called the clump order and be denoted by $K_{i}$.

Figure 2 illustrates the clumping process using an example with $A(t)=7$ particles arriving at a sensor at times 1.9, 5.9, 6.8, 7.5,11.6, 12.8 and $17.1 \mathrm{msec}$ during an observation period of $t=20 \mathrm{msec}$. If particles are assumed to have diameter $4.45 \mathrm{~mm}$ and to be travelling at a constant velocity of $2.225 \mathrm{~mm} / \mathrm{msec}$, the passage time required for each, or deterministic segment length, is $d_{0}=2 \mathrm{msec}$, leading to four clumps of lengths $y_{1}=2, y_{2}=3.6, y_{3}=3.2, y_{4}=2 \mathrm{msec}$ that exit the sensor at times $3.9,9.5,14.8$ and $19.1 \mathrm{msec}$, respectively. Spacings between clumps would be of length $z_{1}=1.9, z_{2}=$ $2.0, z_{3}=2.1$ and $z_{4}=2.3 \mathrm{msec}$ and the four clump orders would be $k_{1}=1, k_{2}=3, k_{3}=2, k_{4}=1$.

The particle clumps constitute a coverage process on one dimension. Hall (1988) describes the process as a simple linear Boolean model; simple because the clump-lengths are line segments and linear because the events occur in one dimension, the time line. Linear Boolean models also arise as linear transects from higher dimensional convex-grain Boolean models. In the language of queueing theory, the number of particles in a clump at the sensor at a given time forms an $M / D / \infty$ queue in the DSL model and an $M / G / \infty$ queue in the RSL model and clump-lengths are called busy periods. There is much literature on these models from queueing theory (Daley (2001)). For statistical inference for the distribution of diameters or more complex quantities describing the grain process, or for Boolean models in higher dimensions, see Molchanov (1997). Handley (1999) derived a discrete approximation to the distribution of clump-length in the linear Boolean model and used it 
for likelihood inference. Crespi et al. (2005) have employed the linear Boolean model for monitoring events of viral activity in human subjects.

\subsection{Likelihood}

Specification of the clump-length density, $f(y ; \lambda)$ is difficult outside of the case where particle diameters are degenerate. In the DSL model, Hall (1988) has shown that the density has point mass $e^{-\lambda t_{0}}$ at $y=t_{0}$, and is otherwise given by

$$
\begin{aligned}
& f\left(y ; \lambda, t_{0}\right)=\lambda \frac{e^{-\lambda t_{0}}}{1-e^{-\lambda t_{0}}} \\
& {\left[1+\sum_{j=1}^{s-1} \frac{(-1)^{j}}{j !}\left\{\lambda\left(y-(j+1) t_{0}\right)\right\}^{j-1} e^{-j \lambda t_{0}}\left\{\lambda\left(y-(j+1) t_{0}\right)+j\right\}\right]}
\end{aligned}
$$

where $y>t_{0}$ and $s$ is the largest integer such that $t_{0}<y / s$. The continuous part of the density is uniform over $\left(t_{0}, 2 t_{0}\right)$, and decreasing for $y>2 t_{0}$. For small $\lambda, f\left(y ; \lambda, t_{0}\right)$ can be approximated by the uniform distribution on $\left(t_{0}, 2 t_{0}\right)$, for large $\lambda$ it can be approximated by an exponential distribution. The fitted density $f\left(y ; \lambda=0.40, t_{0}=2.00 \mathrm{msec}\right)$ overlays the probability histogram of experimental clump-lengths in Figure 1.

For RSL models, likelihood inference is difficult because of the complexity of the clump-length distributions (Handley (2004)). In the DSL model, an approximate likelihood function can be specified by ignoring the residual lifetime of the process. The residual lifetime is the duration of the last incomplete clump or spacing. A clump is a singleton if there are no arrivals within $t_{0}$ time units of the start of the clump, an event which occurs with probability $e^{-\lambda t_{0}}$. Let $M_{1}=\#\left\{y_{i}: y_{i}=t_{0}\right\}$ denote the number of singleton clumps. By independence of clump-lengths, the approximate partial Boolean likelihood can be factored into components for singleton point masses and multi-particle clump-length densities:

$$
\tilde{\mathcal{L}}\left(\lambda ; y_{1}, \ldots, y_{N(t)}\right)=\underbrace{e^{-m_{1} \lambda t_{0}}}_{\text {singletons }} \underbrace{\prod_{i: y_{i}>t_{0}} f\left(y_{i} ; \lambda, t_{0}\right)}_{\text {multi-particle lengths. }}
$$

Spacings $z_{1}, z_{2}, \ldots$ are not available for the experiments analyzed in section 4 . For cases where the $z_{i}$ are available, an approximate complete Boolean likelihood may be obtained by multiplying the partial likelihood by the likelihood from an exponential random sample, $\lambda^{N} e^{-\lambda \sum z_{i}}$.

For large $t$, the maximum likelihood estimator of $\lambda$ based on $\tilde{\mathcal{L}}$ is approximately normally distributed. However, the analytic expression for the Fisher information is unwieldy, particularly for large clump-lengths, where the degree of the polynomial components of the clump-length density is high. Alternatively, approximate confidence regions can be constructed from the likelihood ratio test statistic, which has an approximate $\chi^{2}$ distribution on 1 degree of freedom.

In the RSL model, where segment lengths are distributed as a random sample from a known distribution with distribution function $G(x ; \theta)$, the clump-length density and resulting likelihood are considerably more complex. Let $f_{R S L}(y ; \lambda, \theta)$ denote the clump-length density, which depends on the unknown parameters, $\lambda$ and $\theta$. Ignoring the residual lifetime, the partial likelihood of the complete clumps is then

$$
\tilde{\mathcal{L}}_{R S L}\left(\lambda, \theta ; y_{1}, \ldots, y_{N(t)}\right)=\lambda^{N} e^{-\lambda \sum z_{i}} \prod_{i=1}^{N(t)} f_{R S L}\left(y_{i} ; \lambda, \theta\right) .
$$


Hall (1988) shows that the Laplace transform $\gamma$ of $Y$ is

$$
\gamma(s)=1+\frac{s}{\lambda}-\left(\lambda \int_{0}^{\infty} \exp \left\{-s t-\lambda \int_{0}^{t}\{1-G(x ; \theta)\} d x\right\} d t\right)^{-1} .
$$

Stadje (1985) obtains the clump-length distribution function $F_{R S L}(y)$ by inversion of $\gamma$, but it is an infinite sum of self-convolutions of a function that may involve an integral with no analytic solution, making inference based on $\tilde{\mathcal{L}}_{R S L}$ difficult.

\subsection{M-estimation}

An important issue in estimation of $\lambda$ is robustness under model misspecification. Inspection of the clump-lengths from the experimental data, such as the run depicted in Figure 1, reveals that the number of clumps with lengths slightly in excess of $t_{0}$ is greater than expected, so that the distribution between $t_{0}$ and $2 t_{0}$ is not uniform. This can be caused by variability in diameter or velocity or by errors of measurement. A desirable property for any estimator is robustness to this departure from model assumptions.

For mean segment length $\mu$, the mean clump-length is given by

$$
E(Y ; \lambda)=\frac{e^{\lambda \mu}-1}{\lambda}
$$

in either the DSL or RSL model, regardless of the distribution of segment lengths (Hall (1988)). For known $\mu$, consider the $M$-estimator $\tilde{\lambda}$ which satisfies

$$
\bar{y}=\frac{e^{\tilde{\lambda} \mu}-1}{\tilde{\lambda}} .
$$

A solution exists by the mean value theorem with $E(Y ; \lambda)$ increasing in $\lambda$. Though there is no analytic solution, the equation can be solved rapidly using any root-finding procedure, such as the uniroot function in the $R$ statistical software package (Ihaka and Gentleman (1996)). A starting point that works in simulations is given by $\tilde{\lambda}=(\bar{y}-\mu) /\left(2 \mu^{2}\right)$, which is the solution obtained using a second order expansion of $e^{\tilde{\lambda} \mu}$ about 0 . An interesting aspect of the sampling distribution of $\tilde{\lambda}$ is that it is negative whenever $\bar{y}<\mu$, an event whose probability is small as long as $\lambda \mu$ is not too small.

This estimating equation for $\lambda$ can be written

$$
\sum_{i} \psi\left(y_{i}, \lambda\right)=0
$$

where $\psi(y, \lambda)=y-\lambda^{-1}\left(e^{\lambda \delta}-1\right)$. Large-sample theory for $M$-estimators, (see, e.g. Stefanski and Boos (2002)) can be used for inference about $\lambda$. For a random sample of $n$ clump-lengths $y_{1}, \ldots, y_{n}$, the asymptotic distribution of $\tilde{\lambda}$ is given by

$$
\sqrt{n}(\tilde{\lambda}-\lambda) \stackrel{\mathcal{L}}{\longrightarrow} N\left(0, C / B^{2}\right)
$$

where $B$ and $C$ are functions of $\lambda$ defined by

$$
\begin{aligned}
& B(\lambda)=E\left(-\frac{\partial}{\partial \lambda} \psi\left(Y_{1}, \lambda\right)\right) \\
& C(\lambda)=E\left(\psi^{2}\left(Y_{1}, \lambda\right)\right) .
\end{aligned}
$$


Since $\psi$ is linear in $Y$, the expectation operations are straightforward:

$$
\begin{aligned}
& B(\lambda)=\frac{e^{\lambda \mu}(\lambda \mu-1)+1}{\lambda^{2}} \\
& C(\lambda)=\operatorname{Var}(Y ; \lambda) .
\end{aligned}
$$

The variance of $Y$ depends on the distribution of segment lengths. In the DSL model with $t_{0}=\mu$,

$$
\operatorname{Var}(Y)=\lambda^{-2}\left(e^{2 \lambda \mu}-2 \lambda \mu e^{\lambda \mu}-1\right) .
$$

In the RSL model with segment lengths distributed according to the general distribution function $G(x)$, clump-lengths have variance

$$
\operatorname{Var}(Y)=2 \lambda^{-1} e^{\lambda \mu} \int_{0}^{\infty}\left(\exp \left[\lambda \int_{t}^{\infty}(1-G(x)) d x\right]-1\right) d t-\lambda^{-2}\left(e^{\lambda \mu}-1\right)^{2}
$$

which can be estimated using the sample variance of clump-lengths, $s_{y}^{2}$. Estimators for the variance of $\tilde{\lambda}$ are then given by

$$
\widehat{\operatorname{Var}}(\tilde{\lambda})=n^{-1} \frac{\tilde{\lambda}^{2}\left(e^{2 \tilde{\lambda} \mu}-2 \tilde{\lambda} \mu e^{\tilde{\lambda} \mu}-1\right)}{\left(e^{\tilde{\lambda} \mu}(\tilde{\lambda} \mu-1)+1\right)^{2}}
$$

in the DSL model and

$$
\widehat{\operatorname{Var}}_{\mathrm{G}}(\tilde{\lambda})=n^{-1} \frac{\tilde{\lambda}^{4} s_{y}^{2}}{\left(e^{\tilde{\lambda} \mu}(\tilde{\lambda} \mu-1)+1\right)^{2}}
$$

in either the DSL or RSL model. In large samples, approximate confidence intervals for $\lambda$ can be constructed from these estimates along with the normal approximation for $\tilde{\lambda}$.

\subsection{Other estimators}

For the DSL model with common deterministic passage time $t_{0}$, other method-of-moments (MOM) estimators can be constructed using only the clumpcount $(N(t))$ and singleton count $\left(M_{1}\right)$ statistics. The sequence of i.i.d. sums $\left\{Z_{i}+Y_{i}\right\}$ is a renewal process. Elementary renewal theory $(\operatorname{Cox}(1962))$ yields that as $t \rightarrow \infty$,

$$
\frac{N(t)-t / \mu_{R}}{\sigma_{R} \sqrt{t / \mu_{R}^{3}}} \stackrel{\mathcal{L}}{\longrightarrow} N(0,1)
$$

where $\mu_{R}$ and $\sigma_{R}^{2}$ denote the mean and variance of a randomly sampled renewal period. In DSL model with deterministic common passage time $t_{0}$,

$$
\begin{array}{llcc}
\mu_{R}=E(Z+Y) & = & \lambda^{-1} e^{\lambda t_{0}} \\
\sigma_{R}^{2}=\operatorname{Var}(Z+Y) & = & \lambda^{-2}\left(e^{2 \lambda t_{0}}-2 \lambda t_{0} e^{\lambda t_{0}}\right) .
\end{array}
$$

Moments for $N(t)$ are then

$$
\begin{aligned}
E[N(t)] & \approx \lambda t e^{-\lambda t_{0}} \\
\operatorname{Var}[N(t)] & \approx \lambda t\left(e^{-\lambda t_{0}}-2 \lambda t_{0} e^{-2 \lambda t_{0}}\right) .
\end{aligned}
$$

The probability that a randomly selected clump is a singleton is $e^{-\lambda t_{0}}$ so that $E\left(M_{1}\right)=\lambda t e^{-2 \lambda t_{0}}$. A MOM estimator based on the observed number of singletons is then

$$
\tilde{\lambda}_{S}=-\frac{1}{t_{0}} \log \left(\frac{M_{1}}{N(t)}\right)
$$


Grift et al. (2001) and Grift (2002) base estimation of total mass flow on this estimator. Other estimators of $\lambda$ can be constructed by consideration of vacancy, $V \approx \sum Z_{i}$, or total time that that the sensor is unoccupied. Hall (1988) develops asymptotic theory for a number of vacancy-based estimators. Measurements of $V$ were not available from the experiments discussed in section 4 , and vacancy-based estimators are not considered further.

\subsection{Simulation}

Simulations were undertaken to provide some information about the performance of these estimators, with three goals in particular: a comparison of the efficiency of the moment estimator $\tilde{\lambda}$ relative to the MLE under the DSL model, an investigation of the robustness of the MLE under the RSL model and a comparison of coverage probabilities of confidence intervals resulting from the two variance estimates of the asymptotically normal $M$-estimator, $\tilde{\lambda}$. Particle arrivals were generated according to a Poisson process. Three cases with an increasing degree of clumping were simulated using flow intensities of $\lambda=0.1,0.2$ and 0.3 . Two times were considered for the length of the total observation period, $t=1000$ and $t=10000$. Preliminary experiments with particles far enough apart so that there was no clumping indicated that measured passage times were normally distributed. So, passage times for individual particles were generated from a normal distribution with a mean of $\mu=5$ with three different standard deviations, $\sigma=0,0.5,1$. The first of these standard deviations leads to the DSL model, the others to RSL models. The approximate mean clump counts for the DSL model were $E[K] \approx 1.6,2.7,4.5$ for the three flow rates, $\lambda=0.1,0.2,0.3$, respectively. The simulation experiment then had a crossed $3 \times 2 \times 3$ design, with $n=500$ independent datasets generated per combination of $\lambda, t$ and $\sigma$. Normal plots and Kolmogorov-Smirnov statistics did not indicate any obvious non-normality for either the MLE or $\tilde{\lambda}$.

Table 1 summarizes the results of the simulation. The bias of the $M$-estimate relative to $\lambda$ and the efficiency relative to the MLE are given in the middle section. Though the bias of the MLE formulated under the DSL model dissipates with increasing $\lambda$ or $t$, it does not exhibit robustness to heterogenous segment lengths, in the sense that it has larger variance than the $M$-estimate. Empirical coverage probabilities for $95 \%$ confidence intervals based on the LRT and those of the form $\tilde{\lambda} \pm 1.96 S E$ where $S E$ denotes the appropriate estimated asymptotic standard error from Section 2 are given in the right section of Table 1 . For the shorter simulations $(t=1000)$, there is a tendency for coverage probabilities based on $\tilde{\lambda}$ to be low. For datasets with a larger number of clumps $(t=10000)$, the nominal coverages for intervals based on $\tilde{\lambda}$ are reached. With $n=500$ simulations, the Monte Carlo standard error is such that any sample proportion less than 0.934 is significantly less than the nominal 0.95 with comparisonwise error rate 0.05 . Additionally, the intervals around the $M$-estimate that use the standard error, $S E_{G}$, which is a function of the sample variance of the clump-lengths, appear to do better for the RSL models with large $N(t)$, particularly for the noisy segment length $\sigma=1$ case. The likelihood ratio interval gives coverages consistent with nominal levels in simulations with the DSL model, but breaks down under the RSL model where the likelihood is misspecified. In summary, the recommendation based on these simulations is that the $M$-estimator is reasonably efficient under the DSL model and robust to the conditions of the RSL model. Confidence intervals based on the standard error $S E_{G}$ meet nominal coverage probabilities in large samples under either model.

\section{Estimation of total particle flow}

In the case where either $\lambda$ is known or variance in its estimation is negligible, total particle flow may be estimated by $E[A(t)]=\lambda t$. When $t$ is not available, another estimator can be formed by substitution of $t \approx \sum Y_{i}+\sum E\left(Z_{i}\right)$ into the expression giving $E[\widehat{A(t)}]=\lambda \sum Y_{i}+N(t)$. 
Table 1. Simulation: relative efficiency and coverage probability of $\lambda$ estimators

\begin{tabular}{ccccccccc}
\hline \multicolumn{3}{c}{ Parameters } & \multicolumn{3}{c}{ Rel. } & \multicolumn{3}{c}{ Rel. } \\
$\sigma$ & $t$ & $\lambda$ & $N(t)$ & Bias & Eff. & LRT & $S E(\tilde{\lambda})$ & $S E_{G}(\tilde{\lambda})$ \\
\hline 0 & 1000 & 0.1 & 60.1 & 0.01 & 0.89 & 0.966 & 0.950 & 0.944 \\
0 & 1000 & 0.2 & 73.2 & -0.01 & 0.95 & 0.956 & 0.956 & 0.934 \\
0 & 1000 & 0.3 & 66.7 & -0.01 & 0.98 & 0.948 & 0.946 & 0.944 \\
0 & 10000 & 0.1 & 605.8 & 0.00 & 0.83 & 0.940 & 0.942 & 0.942 \\
0 & 10000 & 0.2 & 734.5 & 0.00 & 0.88 & 0.960 & 0.956 & 0.950 \\
0 & 10000 & 0.3 & 669.6 & 0.00 & 0.97 & 0.942 & 0.938 & 0.942 \\
\hline 0.5 & 1000 & 0.1 & 60.2 & 0.56 & 8.3 & 0.128 & 0.922 & 0.916 \\
0.5 & 1000 & 0.2 & 73.0 & 0.15 & 2.2 & 0.768 & 0.910 & 0.902 \\
0.5 & 1000 & 0.3 & 66.3 & 0.05 & 1.1 & 0.940 & 0.952 & 0.950 \\
0.5 & 10000 & 0.1 & 606.1 & 0.57 & 82.4 & 0.000 & 0.942 & 0.946 \\
0.5 & 10000 & 0.2 & 736.1 & 0.15 & 17.2 & 0.002 & 0.946 & 0.954 \\
0.5 & 10000 & 0.3 & 670.1 & 0.05 & 3.3 & 0.646 & 0.938 & 0.940 \\
\hline 1 & 1000 & 0.1 & 60.5 & 0.56 & 7.3 & 0.136 & 0.914 & 0.940 \\
1 & 1000 & 0.2 & 73.5 & 0.13 & 1.9 & 0.798 & 0.922 & 0.920 \\
1 & 1000 & 0.3 & 66.9 & 0.03 & 0.98 & 0.952 & 0.944 & 0.942 \\
1 & 10000 & 0.1 & 605.3 & 0.56 & 71.9 & 0.000 & 0.922 & 0.952 \\
1 & 10000 & 0.2 & 735.7 & 0.14 & 13.6 & 0.016 & 0.924 & 0.946 \\
1 & 10000 & 0.3 & 668.6 & 0.04 & 3.0 & 0.694 & 0.944 & 0.954 \\
\hline
\end{tabular}

In the DSL model, clump orders $\left(K_{1}, K_{2}, \ldots\right)$ may be shown (Pippenger (1998)) to be geometrically distributed. A clump is of order one $\left(K_{i}=1\right)$ if there are no arrivals within $t_{0}$ time units of the start of the clump, which occurs with probability $e^{-\lambda t_{0}}$. A clump is of order two if there is exactly 1 arrival within $t_{0}$ units and none in the next $t_{0}$ time units, an event which occurs with probability $\left(1-e^{-\lambda t_{0}}\right) e^{-\lambda t_{0}}$ and so on. $K_{1}, K_{2}, \ldots$ are then independent geometric random variables with support on positive integers:

$$
\operatorname{Pr}\left(K_{i}=k\right)=\left(1-e^{-\lambda t_{0}}\right)^{k-1} e^{-\lambda t_{0}} \quad \text { for } k=1,2, \ldots
$$

with $E\left(K_{i}\right)=e^{\lambda t_{0}}$ and $\operatorname{Var}\left(K_{i}\right)=e^{2 \lambda t_{0}}-e^{\lambda t_{0}}$. If the system is vacant when observation ends at time $t$, then total particle flow may be expressed as the sum of these clump orders: $A(t)=K_{1}+\cdots+K_{N(t)}$. If the system is occupied at time $t$, there is a partial clump that contributes a relatively small amount of particle flow for large $t$. Expressing total particle flow $A(t)$ as the sum of clump orders each with mean $e^{\lambda t_{0}}$ suggests the estimator $\hat{A}_{1}(t ; \lambda)=N(t) e^{\lambda t_{0}}$. When evaluated at the $M$-estimator $\tilde{\lambda}$, with mean passage time $\mu=t_{0}$, the two estimators of total particle flow become equivalent: $\tilde{A}_{0}(\tilde{\lambda})=N(t) e^{\tilde{\lambda} \mu}=\hat{A}_{1}(t ; \tilde{\lambda})$.

More efficiency might be gained by conditioning on the clump lengths. The estimator $p(y)$ of an individual clump order which is a function of the clump length $y$ and minimizes the mean squared error $E\left[(K-p(y))^{2}\right]$, is the Bayes estimate, or $p(y)=E(K \mid Y=y)$. An estimate of mean total particle flow $E[A(t)]=E\left[\sum K_{i}\right]$ is then given by summing over clumps:

$$
\hat{A}_{B}(t ; \lambda)=\sum_{i=1}^{N(t)} E\left(K_{i} \mid Y_{i} ; \lambda\right) .
$$

Of course $E\left[K_{i} \mid Y_{i}=t_{0} ; \lambda\right]=1$. The approach used by Hall (1988) to derive the clump length density $f(y)$ in the DSL model may be extended to obtain the conditional mean of clump orders, $E(K \mid Y)$. Let the beginning of a clump be the origin and let $k$ denote an integer greater than unity. The joint event $K=k$ and $Y \in(y, y+d y)$ occurs if and only if there is a particle arrival at $\left(y-t_{0}, y-t_{0}+\Delta y\right)$, 
no arrival in $\left(y-t_{0}+\Delta y, y\right)$, exactly $k-2$ arrivals in $\left(0, y-t_{0}\right)$, and the nearest neighbor of each of these $k-2$ arrival times is not further than $t_{0}$ time units away. Since the first three of these conditions are independent and the fourth is conditionally independent of the first two given the third, the joint probability of these four events is the product

$$
\lambda \Delta y e^{-\lambda t_{0}} \frac{\left(\lambda\left(y-t_{0}\right)\right)^{k-2}}{(k-2) !} e^{-\lambda\left(y-t_{0}\right)} p_{k-2}\left(\frac{t_{0}}{y-t_{0}}\right)
$$

where $p_{n}(u)$ denotes the chance that the largest division formed by a random sample of $n$ points taken from the unit interval does not exceed $u$. This probability is given by

$$
\begin{aligned}
p_{n}(u) & =\sum_{j=0}^{\left[u^{-1}\right]}(-1)^{j}\left(\begin{array}{c}
n+1 \\
j
\end{array}\right)(1-j u)^{n} \\
& =1-(n+1)(1-u)^{n}+\left(\begin{array}{c}
n+1 \\
2
\end{array}\right)(1-2 u)^{n}-\ldots
\end{aligned}
$$

where [.] denotes the largest integer not exceeding the argument. Division by $f(y)$ and differentiation with respect to $y$ yields the conditional density

$$
\operatorname{Pr}(K=k \mid Y=y)=\frac{\lambda e^{-\lambda y}}{f(y)} \frac{\left(\lambda\left(y-t_{0}\right)\right)^{k-2}}{(k-2) !} p_{k-2}\left(\frac{t_{0}}{y-t_{0}}\right) .
$$

If $s=\left[y / t_{0}\right]$, then summation over positive integers yields an exact expression for the conditional mean:

$$
\begin{aligned}
E(K \mid Y=y) & =\sum_{k=s+1}^{\infty} k \operatorname{Pr}(K=k \mid Y=y) \\
& =\frac{\lambda e^{-\lambda t_{0}}}{f(y)} \sum_{k=s+1}^{\infty} k \frac{\left(\lambda\left(y-t_{0}\right)\right)^{k-2}}{(k-2) !} p_{k-2}\left(\frac{t_{0}}{y-t_{0}}\right) \\
& =\frac{\lambda e^{-\lambda t_{0}}}{f(y)} \sum_{k=s+1}^{\infty} k \frac{\left(\lambda\left(y-t_{0}\right)\right)^{k-2}}{(k-2) !} \sum_{j=0}^{s-1}(-1)^{j}\left(\begin{array}{c}
k-1 \\
j
\end{array}\right)\left(1-\frac{j t_{0}}{y-t_{0}}\right)^{k-2}
\end{aligned}
$$

Inspection of $\operatorname{Pr}(K=k \mid Y=y ; \lambda)$ reveals that for $t_{0}<y<2 t_{0}, K$ has the translated Poisson distribution with mean and variance that are linear in $y$. For larger $y$, numerical evaluation of $E(K \mid Y=y)$ be difficult. Inspection of plots for larger $y$ and various values of $\lambda$ indicates that after a jump discontinuity of $\lambda t_{0} e^{-\lambda t_{0}}\left(1-e^{-\lambda t_{0}}\right)^{-1}$ at $y=2 t_{0}$, approximate linearity extends to $y>2 t_{0}$. For cases where $N(t)$ is large and there is heavy clumping, $E(K \mid Y=y)$ can be approximated by linear interpolation to save computational effort.

\subsection{Simulation}

The performances of these estimators of mean total particle flow are compared using the simulated data from section 2. Error for either $\hat{A}_{1}$ or $\hat{A}_{B}$, as a percentage of the mean particle flow is assessed using the relative root mean squared error, RRMSE:

$$
\operatorname{RRMSE}(\hat{A}(t))=\frac{1}{\overline{A(t)}} \sqrt{500^{-1} \sum_{i}\left(\hat{A}_{i}(t)-A_{i}(t)\right)^{2}}
$$

where $i$ indexes the 500 simulated datasets. Table 2 summarizes relative bias and RRMSE of estimates obtained by substitution of the $M$-estimates $\tilde{\lambda}$ into the expressions $\hat{A}_{1}(t)=N(t) e^{\lambda \mu_{t}}$ and 
Table 2. Error in estimation of total particle flow from simulations.

\begin{tabular}{ccccccc}
\multicolumn{3}{c}{ Parameters } & \multicolumn{2}{c}{ Relative bias } & \multicolumn{2}{c}{ RRMSE } \\
$\sigma_{t}$ & $t$ & $\lambda$ & $\hat{A}_{1}$ & $\hat{A}_{B}$ & $\hat{A}_{1}$ & $\hat{A}_{B}$ \\
\hline 0 & 1000 & 0.1 & -0.008 & -0.007 & 0.042 & 0.033 \\
0 & 1000 & 0.2 & -0.012 & -0.011 & 0.048 & 0.045 \\
0 & 1000 & 0.3 & -0.014 & -0.013 & 0.049 & 0.048 \\
0 & 10000 & 0.1 & -0.001 & -0.001 & 0.014 & 0.01 \\
0 & 10000 & 0.2 & 0.000 & 0.000 & 0.015 & 0.014 \\
0 & 10000 & 0.3 & -0.002 & -0.002 & 0.016 & 0.015 \\
\hline 0.5 & 1000 & 0.1 & -0.003 & 0.178 & 0.048 & 0.185 \\
0.5 & 1000 & 0.2 & -0.008 & 0.061 & 0.051 & 0.076 \\
0.5 & 1000 & 0.3 & -0.011 & 0.013 & 0.054 & 0.052 \\
0.5 & 10000 & 0.1 & 0.000 & 0.184 & 0.014 & 0.184 \\
0.5 & 10000 & 0.2 & -0.001 & 0.067 & 0.015 & 0.068 \\
0.5 & 10000 & 0.3 & -0.003 & 0.022 & 0.015 & 0.026 \\
\hline 1 & 1000 & 0.1 & -0.002 & 0.189 & 0.058 & 0.198 \\
1 & 1000 & 0.2 & -0.010 & 0.063 & 0.053 & 0.079 \\
1 & 1000 & 0.3 & -0.011 & 0.018 & 0.052 & 0.052 \\
1 & 10000 & 0.1 & 0.001 & 0.192 & 0.018 & 0.193 \\
1 & 10000 & 0.2 & 0.000 & 0.072 & 0.017 & 0.074 \\
1 & 10000 & 0.3 & -0.001 & 0.026 & 0.016 & 0.031 \\
\hline
\end{tabular}

$\hat{A}_{B}(t ; \lambda)$ for each simulated experimental condition. The estimation based on clumpwise estimated clump orders $\hat{A}_{B}$, is competitive under the DSL model $\left(\sigma_{t}=0\right)$ for smaller sample sizes, $(t=1000)$. It suffers from some positive bias in RSL models that appears to decrease as flow rate $\lambda$ increases, though it remains inferior to $\hat{A}_{1}$ despite smaller variance and higher correlation with $A(t)$. In the RSL model, many singleton clumps have clump lengths slightly in excess of the mean singleton passage time $\mu_{t}$ and so have estimated orders in excess of 1 . This may lead to a positive bias for the clumpwise estimators which is particularly acute when support is high near $Y=\mu_{t}$. This theory is supported by the poor performance under light clumping, when $\lambda=0.1$ and density near $Y=\mu_{t}$ is highest among values of $\lambda$ considered in the simulation.

In summary, for minimal relative error, these simulations suggest the use of the simple $\hat{A}_{1}(t)$ estimator, which is unbiased and involves less computation than the clumpwise estimator $\hat{A}_{B}(t)$. A slight loss of efficiency under the DSL model may be offset by the superior performance in the RSL model. Expressed relative to total particle flow, the root MSE was not larger than $5.8 \%$ in any of the conditions simulated here.

\section{Experimental data}

An optical sensor was used to measure clump lengths and clump velocities in experiments (Grift et al. (2001); Grift (2002)) in which a known number of spherical particles was dropped through a device simulating an aerial spreader duct. Various quantities of several kinds of particles (BBs, urea fertilizer) were dropped at several velocities. The data considered here include 10 runs with 4000 identical steel particles (BBs) dropped from each of two heights and 5 runs with 2000 BBs dropped from a fixed height. Mean $(\bar{y})$ and variance $\left(s_{y}^{2}\right)$ of physical lengths (in $m m$ ) appear in Table 3 along with other statistics from the experiments. Division by mean velocity $(\bar{v}=2.23 \mathrm{~mm} / \mathrm{msec})$ was used to transform the measurements to the time line (in $\mathrm{msec}$ ) to obtain Figure 1. In general, velocity was reasonably constant within a run of the experiment.

The data were imperfect and some outlier removal was undertaken. For example, the counter returned several clumps with negative velocities or negative physical lengths, or sometimes both. 
Table 3. Estimation from experiments with BBs

\begin{tabular}{ccccccc}
\hline Run & $N$ & $\bar{y}$ & $s_{y}^{2}$ & $\hat{\lambda}(S E)$ & $\hat{A}_{1}$ & $\hat{A}_{B}$ \\
\hline 1 & 2958 & 5.22 & 3.07 & $0.070(0.003)$ & 4041 & 4921 \\
2 & 2930 & 5.22 & 2.91 & $0.070(0.003)$ & 3997 & 4946 \\
3 & 2891 & 5.26 & 3.39 & $0.073(0.003)$ & 4008 & 4874 \\
4 & 2935 & 5.22 & 3.00 & $0.070(0.003)$ & 4000 & 4944 \\
5 & 2990 & 5.16 & 2.88 & $0.065(0.003)$ & 3986 & 4941 \\
6 & 2941 & 5.20 & 3.00 & $0.068(0.003)$ & 3984 & 4883 \\
7 & 2983 & 5.15 & 2.84 & $0.064(0.003)$ & 3969 & 4900 \\
8 & 2956 & 5.16 & 2.90 & $0.065(0.003)$ & 3952 & 4846 \\
9 & 2894 & 5.24 & 3.12 & $0.071(0.003)$ & 3976 & 4831 \\
10 & 2914 & 5.25 & 3.11 & $0.073(0.003)$ & 4025 & 4931 \\
\hline 11 & 1821 & 6.76 & 11.77 & $0.176(0.005)$ & 3988 & 4299 \\
12 & 1770 & 6.85 & 11.56 & $0.182(0.005)$ & 3976 & 4303 \\
13 & 1805 & 6.80 & 12.30 & $0.179(0.005)$ & 4000 & 4321 \\
14 & 1748 & 6.96 & 12.57 & $0.188(0.005)$ & 4038 & 4333 \\
15 & 1800 & 6.85 & 12.13 & $0.182(0.005)$ & 4040 & 4340 \\
16 & 1784 & 6.93 & 14.82 & $0.186(0.005)$ & 4089 & 4403 \\
17 & 1772 & 6.93 & 12.56 & $0.187(0.005)$ & 4064 & 4341 \\
18 & 1788 & 6.89 & 13.28 & $0.184(0.005)$ & 4052 & 4346 \\
19 & 1812 & 6.78 & 12.01 & $0.178(0.005)$ & 3995 & 4317 \\
20 & 1790 & 6.84 & 11.98 & $0.181(0.005)$ & 4005 & 4330 \\
\hline 21 & 746 & 7.54 & 17.10 & $0.219(0.008)$ & 1981 & 2143 \\
22 & 791 & 7.24 & 13.20 & $0.204(0.007)$ & 1959 & 2141 \\
23 & 777 & 7.46 & 13.15 & $0.215(0.007)$ & 2024 & 2184 \\
24 & 774 & 7.30 & 13.23 & $0.207(0.007)$ & 1941 & 2102 \\
25 & 745 & 7.57 & 13.39 & $0.221(0.007)$ & 1989 & 2133 \\
\hline & & & & & & \\
& & & & &
\end{tabular}

Additionally, each run contained a very small number of extremely short clumps, much less than the particle diameter, possibly due to matter other than the particles of interest blocking the sensor. The number of questionable clump measurements that were removed did not exceed $1 \%$ for any of the 25 runs.

In these experiments, total particle flow is fixed and total flow time varies with run and is not observed. The opposite is true for the application of mass flow measurement during aerial application of fertilizer particles. The theoretical results regarding inference for the random particle flow $A(t)$ for fixed $t$ do not necessarily hold under the conditions of the experiment, where $A(t)$ is fixed and $t$ varies and is not observed. However, Table 3 provides some indication that estimates for total particle flow, $A(t)$, have good empirical performance when it is treated as random, at least in these experiments.

The observed value of the estimator $\hat{A}_{1}$ is given in the penultimate column. It appears to perform reasonably well under these conditions. The average of $\hat{A}_{1}$ over runs 1-20 is 9 and the root mean squared error from 4000 is 35.5 , which is $0.9 \%$ of the target. There is some evidence of positive bias in the high intensity runs 11-20. A two-sided $t$-test of the hypothesis that $E\left[\hat{A}_{1}\right]=4000$ under the conditions of runs 11-20 yielded a $p$-value of 0.065 on $d f=9$.

Higher flow rates lead to more clumps per particle, fewer singletons, and larger variance in estimation of clump order, either conditionally as in $\hat{A}_{B}$ or unconditionally, as in $\hat{A}_{1}$. The standard deviations of $\hat{A}_{1}$ under the light (runs 1-10) and heavy (runs 11-20) clumping conditions with 4000 BBs were $s_{l}=26.4$ and $s_{h}=37.1$, respectively. The estimates $\hat{A}_{B}$, which are based upon the DSL model, exhibit substantial positive bias, as they did for data simulated under the RSL model. The same is true for the MLE of $\lambda$.

To assess the goodness of fit of the linear Boolean models, probability histograms of the clump 
length data were checked for agreement with the estimated density $f(y ; \tilde{\lambda}, d)$. One such check appears in Figure 1, which exhibits reasonable fit except for slightly lowered mass at the mean segment length, $\mu=2 \mathrm{msec}$ and slightly more observations just above the mean segment length than expected under uniformity of this part of the density. All of the other histograms exhibited the same three distinctive features of a spike near this fixed segment length, near uniformity between one and two of these lengths and a long right tail. Quantile plots and Kolmogorov-Smirnov goodness-of-fit tests, for estimates in runs 1-10 or runs 11-20 do not indicate any non-normality in the distribution of $N, \tilde{\lambda}$ or $\hat{A}_{1}$.

\section{Conclusion}

Two versions of a simple linear Boolean model are proposed to describe passage times of clumps of particles in a type II counter system; one assumes deterministically equal passage times for all particles, while the other assumes these to be distributed about a known mean with unknown variance. An $M$-estimator of flow intensity is developed that is intuitively sensible, computationally feasible, and robust to conditions where either particle velocity and/or diameters have substantial variability or are being measured with error by the type II counter.

For total mass flow, $A(t)$, two estimators are developed. The first is simply product of the number of clumps, $N(t)$ and the estimate of the mean number of particles per clump. The second more complex estimator is the clumpwise sum of conditional mean clump orders $(K)$, given clumplengths $(Y)$. In models where segment lengths were deterministically equal, the Bayes estimator exploiting the conditional mean clump order had relative root mean squared error not exceeding $5.0 \%$, and always lower than that of the simpler estimator based only on the $M$-estimate of flow rate and the number of clumps. Under the most favorable conditions, with light particle flow and a long dispersal period, the relative error was as small as $1 \%$. While the Bayes estimator did well in data simulated from the DSL model, it was outperformed by the simpler estimator in simulations where the segment lengths vary according to a normal distribution and in the bench-scale experiments. The relative root mean square error when using the estimator of based ranged between $1.4 \%$ and $5.1 \%$. The relative root mean square errors for the experimental data were $0.6 \%$ and $1.1 \%$, in the low and high intensity runs with $4000 \mathrm{BBs}$, respectively and $1.8 \%$ in the runs with $2000 \mathrm{BBs}$.

\section{References}

Casady, W., H. Downs, and F. Fishel (1997). Calibrating granular pesticide applicators. Technical Report G1273, University of Missouri Extension.

Cox, D. (1962). Renewal Theory. Great Britain: Wiley.

Crespi, C., W. Cumberland, and S. Blower (2005). A queueing model for chronic recurrent conditions under panel observation. Biometrics 61, 193-198.

Daley, D. (2001). The busy period of the $m / g i / \infty$ queue. Queueing Systems 38, 195-204.

Grift, T. (2002). Cluster criteria. Resource: Engineering and Technology for a Sustainable World 9, $9-10$.

Grift, T. and J. Hofstee (1997). Measurement of velocity and diameter of individual fertilizer particles by an optical method. J. of Agric. Eng. Res. 66, 235-238.

Grift, T., J. Walker, and J. Hofstee (2001). Mass flow measurement of granular materials in aerial application - part 2: Experimental model validation. Trans. Am. Soc. Agric. Eng. 44, 27-34. 
Hall, P. G. (1988). Introduction to the Theory of Coverage Processes. U.S.A.: John Wiley \& Sons, Inc.

Handley, J. (1999). Discrete approximation of the linear boolean model of heterogeneous materials. Physical Review E 60, 6150-6152.

Handley, J. (2004). Computationally efficient approximate likelihood procedures for the boolean model. Comput. Statist. and Data Analysis 45, 125-136.

Ihaka, R. and R. Gentleman (1996). R: A language for data analysis and graphics. J. of Comput. and Graphical Statist. 5(3), 299-314.

Molchanov, I. (1997). Statistics of the Boolean Model for Practicioners and Mathematicians. New York: John Wiley \& Sons.

Pippenger, N. (1998). Random interval graphs. Random Structures $\&$ Algorithms 12, 361-380.

Pyke, R. (1958). On renewal processes related to type-i and type-ii counter models. Ann. Math. Statist. 29, 737-754.

Stadje, W. (1985). The busy period of the queueing system $\mathrm{m} / \mathrm{g} / \infty$. J. of Appl. Prob. 22, 697-704.

Stefanski, L. and D. Boos (2002). The calculus of m-estimation. Am. Statist. 56, 29-38. 